\title{
The Buddhist Perspective of Continual Improvement
}

\author{
T. Jayawardane ${ }^{1}$ \& A. C. De Alwis ${ }^{2}$ \\ ${ }^{1}$ Doctoral Student, Faculty of Commerce and Management Studies, \\ University of Kelaniya, Sri Lanka (E-mail: thusharaya@yahoo.com) \\ ${ }^{2}$ Department of Human Resource Management, Faculty of Commerce and Management \\ Studies, University of Kelaniya, Sri Lanka (Email: dealwisac@gmail.com)
}

\begin{abstract}
Continual Improvement (CI) is closely linked to Japanese quality management. As the CI concepts were developed in a strong Buddhist culture in the background, a remarkable similarity can be seen between CI and Buddhism. This paper reveals the overlap between various sciences of CI and various Suttas' in the Sutta Pitaka of Buddhism as the main reference body. Sutta Pitaka is one of the three key branches of Tripitaka which is the oldest and most original text available on the Buddhist philosophy. The similarities that are observed deep inside the tools, techniques and behavioral branches of $C I$ such as cause and effect relations, problem validation, problem solving sequence, problem types, corrective and preventive action, nonconformity, autonomy and knowledge are discussed in detail.
\end{abstract}

Keywords: Buddhism, Continual Improvement, Continuous Improvement, Quality, Total Quality Management

\section{Introduction}

The concept of Continuous Improvement (CI) has emerged with the quality management literature which is closely associated with the Japanese culture and later enriched by and expanded in to many disciplines of management science.

However it should be noted that Japanese management systems were not entirely compatible with other organization cultures. For instance, Yokosawa \& Steenhuis (2012) identifies the issues in transferring Japanese Kaizen concept to other countries; Ngowi (2000) identifies the cultural implications of total quality management implementations.

Secondly, certain recent disclosures on the subject indicate certain behavioral components associated with $\mathrm{CI}$ have not been given proper attention. For instance, Bessant, et al. (2001) states "...much of the literature surrounding CI does not treat the behavioral aspects of the process well". 
Thirdly, some have suggested that continuous improvement methods like obtaining suggestions from employees were visible in the Japanese cultures long before the CI emerge as a concept (Poropat \& Kellett, 2006).

As such, it is only rational to assume there would have been a cultural or behavioral component which is yet to be identified, which leads to the adaptability of CI in certain other cultures. Also it can be assumed that these missing linkages would have evolved through times in the Japanese culture.

Religion plays a role in managerial decision making (Radric, 2007). Japanese culture is largely influenced by Buddhism. However, there is no study conducted so far to correlate the Japanese cultural implications on CI. This paper discusses the influence of Buddhist culture on CI on the assumption that these behavioral and cultural components have emerged through Buddhist teachings which are believed to have shaped and influenced the Japanese way of work for thousands of years.

\section{Continuous Improvements (CI)}

CI represents a series of methods and behaviors which, in combination facilitates organizational transformation towards betterment. However, the existing literature falls short in providing a unique identifier for CI. Bhuiyan \& Baghel (2005) defines $\mathrm{CI}$ as "a culture of sustained improvement targeting the elimination of waste in all systems and processes of an organization"; Radawski (1999) explains CI as a systematic approach to improve an existing product or to introduce a new product. Zangwill \& Kanton (1998) described it as a "technique which has produced substantial improvements..." This ambiguity is basically emerging as a result of identifying CI, by the path by which improvement is obtained or by the result it produced.

"There is considerable and unhelpful confusion in the way the term 'continuous improvement' is used, since it is deployed both as a verb the process whereby a continuous stream of innovations emerge - and also as a noun, referring to the outcome of that process" (Bessant, Caffyn \& Gallagher, 2001).

The purpose of CI can be either survival or growth depending on the organization. As such, some organizations may use CI to reduce waste or simplify their process, whereas in another organization CI deals only with improvement (Bhuiyan \& Baghel, 2005) . Improvement strategies can be in the form of fixing of the broken processes; refining by way of incremental innovations; renovation of the breakthrough improvements or reinvention of the new process (Besterfield, et al., 2003). 
Origins of CI are closely linked with Total Quality Management (TQM) philosophy and the scientific method. CI "is the centerpiece of total quality management" (Ngowi, 2000). Most influential managerial implications of CI can be seen in quality management. By now the CI concepts have broadened enough to be identified as a separate entity of management theory that has influenced many branches of organizational sciences and may as well qualify as an organization culture.

Methodology component of CI is a fact based control and revision algorithm for organizational processes. "This revision in production processes is undertaken by examining data provided from the processes themselves" (Radawski, 1999). The significance of CI lies not only in the managerial control over improvement but also in its underlying belief system which shapes the behavioral component of CI. CI believes that there is never a constant satisfactory level of quality. Poropat \& Kellett (2006) states “...every improvement is just a step on the path of greater control of variation..." In behavioral point of view CI is a continuous learning exercise (Bessant, Caffyn, \& Gallagher, 2001). CI behavior encompasses team work \& innovation which is seen in concepts such as Japanese Jishuken (Marksberry, Badurdeen, Gregory, \& Kreafle, 2010) \& kaizen (Yokosawa \& Steenhuis, 2012).

Ngowi (2000) identifies "corrective action, measurement and goal setting as key CI attributes". The existing control frameworks of CI follows similar scientific pattern which can be observed in quality management; in Deming's Plan-Do-Check-Act (PDCA), Juran's trilogy of planning-control-improvement, European Foundation's Results- Approach-Deployment-Assessment \& Refinement(RADAR), six-sigma's Define-Measure-Analyze-Improve-Control(DMAIC) and Oakland (2004)'s DefineReview-Investigate-Verify-Execute(DRIVE); in project planning in Rettig \& Simon(1993)s' Plan-Approve-Do-Review and Execute (PADRE); in military strategy in Boyd's Observe-Orient-Decide-Act; in health care in Orlando's nursing process in Assessment-Diagnosis-Planning-Implementation -Evaluation(ADPIE).

Broader bifurcation of these frameworks can be made with respect to the positioning of the action component. It can be seen that certain models have given more emphasis to prior to execution. For example the Oakland's DRIVE framework has discussed four stages 1. Define 2.Review 3.Investigate 4.Verify prior to the execution stage, and as a result implied finality of execution. In other words the action component is error-proofed in four stages prior to execution to confirm the final outcome of the action. It should be noted that action phase is also the final stage in the model. Similarly Rettig \& Simons's PADRE \& Boyd's OODA do follow a similar implication. On the other hand Deming's PDCA, RADAR, DMAIC and ADPIE does not imply such finality. It should be noted that the action 
component is in between the beginning and end of the cycle implying existence of post action stages related to improvement.

\section{Comparison of Buddhism and Continuous Improvements}

At face value there is a similarly between CI and Buddhism. The roots of this connection are visible deep inside the evolution of quality management from which CI is conceptualized. This resemblance has provided the Japanese firms with a culture that is favorably responsive to total quality management as against firms in Europe (Poropat \& Kellett, 2006).

Most remarkable similarity between CI and Buddhism is visible in the Four Nobles Truths (FNT). FNT (ariya sacca) is the essence of Dhammacakkappavattana Sutta, the very first teaching of Gautama Buddha .FNT is central to Buddha's teaching (Sumedho, 1992; Piyadassi, 2008; Tsering \& McDougall, 2005).The FNT represent a philosophy behind the cessation of suffering. It describes four truths related to existence and cause of suffering and path to its elimination. FNT's are 1. The noble truth of suffering (dukka); 2. The noble truth of the origin of suffering (samudaya); 3. The noble truth of the cessation of suffering and the origin of suffering (nirodha); 4. The noble truth of the path that leads to the cessation of suffering and the origin of suffering (magga) (Tsering \& McDougall, 2005). In other words the FNT is the realization that "...there is a cause or origin of suffering; there is an end of suffering; and there is path out of suffering..." (Sumedho, 1992).

Use of FNT in management is not entirely new. Application of FNT is observed in a broad range from economics, quality to organizational behavior. Poropat \& Kellett (2006) identifies many fundamental similarities between Buddhism and TQM, "such as both emphasizing empirically-based practices, skepticism about received truth, encouraging continual improvement, and an assumption of change or variation". Fletcher \& Hayes(2005) in describing mindfulness by effects of language and cognition on human action have described FNT in relation to acceptance and commitment therapy and stated that there is a remarkable overlap between principles of acceptance and commitment therapy and Buddhism observed in "...the ubiquitous nature of human suffering and values and committed action". Lamberton (2005) in describing a "version of sustainability that provides clear and unambiguous direction for decision makers" have applied the Buddhist concept of right livelihood to economics, "... leading to an alternative perspective of sustainability". Schumacher (1973) developed a concept of "Buddhist Economics" discussing how the nature of work, labor, production and organization can fundamentally be changed from prevailing macroeconomic perspective, considering various disciplines of Buddhist philosophy. 
FNT describes liberation as emerging from the suffering. In other words the liberation is discussed as inherent to suffering thus making the liberation a path than a process. The cause and effect order of FNT is described below.

Table 01: Cause and Effect

\begin{tabular}{|l|l|l|}
\hline & Result & Casue \\
\hline Problem & Dukka & samudaya \\
\hline Solution & nirodha & magga \\
\hline
\end{tabular}

(Tsering \& McDougall, 2005)

\section{On Past, Present and Future}

The foremost similarity between CI and Buddhism is in acknowledgement of the relationship between actions in past with present results and present actions with future results. Buddhism believes that all current states (of being) are a result of actions of previous states. Devadaha Sutta in Majjima nikaya states that "with the non-doing of new actions, there will be no flow into the future" ${ }^{[1]}$ and credited the existence between past, present and future to actions (karma).Same belief is followed in CI, in expecting a better future state by the present actions. Further, both CI and Buddhism realize the continuation of this action (karma) -result (vipaka) cycle in relation to improvement and suffering respectively. CI believes that "there is never a good-enough level of quality." (Poropat \& Kellett, 2006). Similarly Buddhism believes the perpetual existence of suffering as can be seen in the Visuddhimagga in the wheel of life (samsāracakka). Piyadassi (2008) states "The doctrine of paticca-samuppāda can be illustrated by a circle, for it is the cycle of existence, bhavacakka".

Although a pictorial representation of bhavacakka is not seen in the Theravada school of Buddhism, the Mahayana school of Buddhism from which the Japanese Zen Buddhism is branched describes the cycle of existence or bhavacakka as can be seen painted in Tibetan Buddhist monasteries. It visualizes the fundaments of causes, actions, their linkages, impermanence and the liberation together in a cycle, in different layers. In this context, bhavacakka is a composite of many quality tools such as flow diagram, cause and effect diagram, and is in line with the whole problem solving methodology of quality management.

Deming (1994) described CI as a series of never ending Plan-Do-Check-Act cycles (PDCA), on completion of each, the organization is on an improved state than previous. PDCA also closely resembles to FNT. 


\section{On Cause and Symptoms}

One fundamental doctrine of Buddhism is the principle of cessation. "Buddhism teaches that all compounded things come into being, presently exist, and cease (uppāda, țiti, bhanga)..." (Piyadassi, 2008). It establishes a cause and effect relationship of suffering. Paticcasamuppada (dependent origination) in Samyuktha nikaya describes those arise dependent on causes, cease when the causes are no longer present.

A similar thought process is evident in the CI frameworks in the problem solving context. First, it should be noted that both CI and Buddhism are fundamentally inline in realizing the importance of acting on cause than its symptoms. Throughout the Deming's quality management approach effort to understand the cause and effect relationship in CI perspective is visible. In doing so, mathematical verification was seen obtaining its due recognition. "Only through the statistical verification, according to Deming, can the manager 1) know that he has a problem, and 2)find the problem" (IGNOU, 2001). Oakland (2004) states "In the neverending quest for improvement in the ways processes are operated, numbers and information should always form the basis for understanding, decisions and actions; and a thorough data-gathering, recording and presentation system is essential". As such much emphasis was given to the tools, techniques and systems to analyze and interpret data. In this context $\mathrm{CI}$ is seen as an outcome of record, use and analysis of data and acting on results (Oakland, 2004).

\section{On Problem Types}

Both CI and Buddhism have classified problem types. In CI there are many varieties of problem classification. For instance the design of fishbone diagram has facilitated different types of problems. It is not uncommon to classify the bone structure with the use of other management theories. As such, in manufacturing environments the fishbone can be classified as man, machine, material, method \& measurement $(5 \mathrm{M})$ and in marketing the same can be classified as product, price, place, promotion, people, process, physical evidence(7P) and group the problems along the bone accordingly. However the problem classification of fishbone diagram is not restrained. The problem classification can be user defined in the fishbone diagram. Another management tool that supports problem classifications is the affinity diagram in which a pool of problems is grouped in to user defined classes and levels and their linkages are established. Apart from these, many problem classifications can be seen in quality literature. Besterfield, et al. (2003) classified six problem types as; issues related to compliance, unstructuredness, efficiency, product design and process design. Nikolas (1996) identified three 
problem types as repair, improvement and engineering problems. However it is clear that CI acknowledge multiple types of problems. Yet in Buddhism although there are twelve problem categories explained as twelve nidhana's, it has clearly identified a central problem theme and explained all other problems as originating from one, which is ignorance.

\section{On Knowledge}

Similar emphasis on knowledge is visible with respect to CI in Deming's "system of profound knowledge" (SPK). "Knowledge related to improvement comes from outside" (Deming, 1994). In SPK Deming described four aspects of knowledge supportive to management transformation. SPK is an external visualization of the system in knowledge view point. Appreciating the system thinking, knowledge of the variation, theory of knowledge and knowledge of psychology are components of SPK (Evans, 1996). The SPK have close resembles firstly to Avijja in realization of knowledge as key to success and secondly to FNT in acknowledging several forms of knowledge.

Bessant, et al. (2001) provided a more specific CI framework in relation to knowledge by linking the maturity level of $\mathrm{CI}$ in an organization in to five knowledge categories. At basic level the behavior of CI may be as primitive as "trying out an idea". As the CI process matures it passes the "structured and systematic CI" stage, "strategic CI" stage, "autonomous innovation" stage in order to finally reach at "the learning organization" level. These levels represent different stages of knowledge. Buddhist liberation principle also follows a learning \& realization sequence similar to this approach.

For instance, in pratītyasamutpāda (dependent origination) it is noted that origin of suffering is avijja (ignorance). The learning and avijja relationship is reciprocal as higher the former, lower the latter is. Avijja Sutta in Samyutta Nikaya indicates ignorance as the main cause of all suffering followed by lack of conscience \& lack of concern." In an unknowledgeable person, immersed in ignorance, wrong view arises. In one of wrong view, wrong resolve arises" ${ }^{[2]}$.

\section{On Tools}

Both Buddhism and CI have provided means of arriving at the cause. Ishikawa introduced the seven basic quality tools namely cause and effect diagram, check sheet, control chart, histogram, pareto chart, scatter diagram \& stratification. These tools can be used in stages of problem identification, problem analysis to evaluation of result of actions thus making the quality tools valid throughout the PDCA. However, it should be noted that check sheet, control chart, histogram and scatter 
plot are mostly appropriate in visualizing the symptoms of the current problem. Similarly, cause and effect diagram is more suitable for analysis of causes and pareto chart for management review. "When process improvement starts with careful planning, it results in corrective and preventive actions supported by appropriate quality assurance tools which lead to true process improvement" (Sokovic, Jovanovic, Krivokapic, \& Vujovic, 2009). Similarly, Buddhism in Mahavibhanga Sutta in samyutta Nikaya identifies the Nobel eight-fold path as means of cessation of suffering and also described many tools for cessation of suffering such as five-precepts and eight-precepts.

\section{On Corrective and Preventive Actions}

Buddhism point of view is that all that emerge as a result of action (sanskara), decay from their very nature of emerging, and therefore nothing is permanent. Bodhi (2005), translating from Maha parinibbana Sutta in Digha Niakaya states "Impermanent, alas, are all formations!" As such as all suffering is result of actions (karma); suffering is inherent. Therefore every matter that comes in the purview of karma is temporary, which is also true not only for problems but also for corrective actions as well. In CI literature the corrective actions are the actions that deal with eliminating the re-occurrence of non-conformity. In Buddhism, there are no corrective actions. That is, once the karma has occurred, it will keep on accumulating and resulting in either good or bad suffering. Anguttara Nikaya, Nibbedhika Sutta describes the relationship between suffering and karma valid for past, present and future. Therefore action and suffering follows a cycle which can only be prevented by elimination of the cause; which is karma. The structuring of the process of dependent - origination in FNT follows the same principles followed in preventive action in quality literature as evident in the noble eightfold path. ISO 9000:2008 follows a similar thought process in defining preventive action as "...preventing the occurrence of potential non-conformity".

\section{On Non-conformity}

Buddhism and CI go hand in hand in deciding between conformance and nonconformance. Maha-kammavibhanga sutta in Majjima Nikaya describes linkage between karma that is capable or incapable in producing favorable and unfavorable consequences. A similar pattern is observed in CI literature in recognizing conformance and nonconformance. In toto, the whole structure of the CI process is in line with the karma (action)-vipaka (result) of Buddhism. 


\section{On Vision}

Buddhism and $\mathrm{CI}$ take different views on the final visionary future state. Many academics are affirmative that $\mathrm{CI}$ is a never ending process (Eg: Oakland, 2004; Bhuiyan \& Baghel, 2005; Besterfield, et al., 2003).On the other hand Buddhism takes the view that there is an end to suffering. In Buddhism the third noble truth (nirodha sacca) "...altitude to the ultimate goal of Buddhist practice, nirvana" (Albahari, 2006).

Kotthita sutta in Anguttara Nikaya in addressing what lies beyond nirvana states

"However for the six contact-media go, that is how far complication goes. However far complication goes, that is how far the six contact media go. With the remainderless fading $\&$ stopping of the six contact-media, there comes to be the stopping..."

Most CI tools such as PDCA, RADAR and DRIVE have inherited this never ending attitude of CI. Rettig \& Simons (1993) PADRE project development process is one exception to this. Few similarities exist between PADRE and Buddhism. The first is the realization of the existence of the end of the CI life cycle. PADRE describes the life cycle of a project as a spiral which with each iteration passes Plan-Approve-DoReview and Execute stages. This life cycle ends with the project completion Although PADRE is similar in the context of a $\mathrm{CI}$ in the cyclic aspect with PDCA, it differ in the context of being able to set a completion perspective to the CI lifecycle and in visualizing the repetition of each PADRE as a result of an organized outcome of the previous cycle. Also, PADRE discussed a project life cycle in subcomponent level and emphasis is visible on the need to break down a project in to stages at the initial planning stage itself.

\section{On Problem Solving Sequence}

According to Buddhist classification the cause of suffering is ignorance (avijja). In the absence of actions conditioned by ignorance, there will be liberation, and suffering will cease (Piyadassi, 2008). Digha nikaya Maha nidhana sutta explains the dependency of the co arising of sources of suffering starting from ignorance in a twelve fold sequence. Similarly in in quality management the seven step problem solving methodology describes a sequence of actions in elimination of the problem. However the major difference observed between $\mathrm{CI}$ and Buddhism in their problem solving sequences is in the starting point. Upanisa Sutta in Samyutta nikaya view the twelve fold nidhana(causes) as co-arising; all the twelve nidhana's are a reason for the next in line to exist. Therefore elimination of cause also follow a sequence along the nidhana's, in other words root causes. Thus the starting point of problem 
elimination $\mathrm{n}$ is in the problem itself. On the other hand, the problem solving sequence in quality management follow a pattern similar to PDCA which is qualifies as a controlling sequence of the problem solving process. Here, the starting point of solution is placed external to the problem.

Assutavā Sutta in Samyutta nikaya states "When this is, that is. From the arising of this comes the arising of that. When this isn't, that isn't. From the cessation of this comes the cessation of that". Thus, the elimination of the problem is about eliminating origins in order, to arrive at the final solution; the whole sequence of the twelve fold chain is one stage of the problem. However, the problem solving sequences appearing in CI such as the seven step problem solving methodology does not discuss an elimination order, but rather discuss a path of systematic actions need to be taken to eliminate problems. The seven step continual improvement methodology deals with problem identification, description of the current process(identifying the current situation $\&$ its analysis), arriving at root causes, development of action plans, implementation of solutions, review and learning (CQI Technical Assitance Training Manual, 2010).

It should also be noted that although the problem solving steps does not follow a similar stating point, the tools used in arriving at root causes such as five why analysis follow a same pattern in realizing the co-arising and dependency of the causes. Five-why-analysis is developed in Toyota to go deep in to the bottom of the problem. It is a technique of asking why? Five times in search of an answer to the previous cause with each iteration. (Ohno, 1988). Used in conjunction with the cause and effect diagram, it enables to visualize a sequence of causes.

\section{On Realization of Opportunities}

Another important similarity is in the self-realization perspective of solutions in Buddhism and CI. Both have emphasized on ones' own personal reasoning in support of judgment. Kalama sutta in Angutthra Nikaya mentions not to "go by reports, by legends, by traditions, by scripture, by logical conjecture, by inference, by analogies, by agreement through pondering views, by probability, or by the thought" but emphasized on free inquiry; "when you know for yourself". In Toyota, Ohno deploy a similar approach consisting of three realities. It's a go and see "Genbutsu" approach for problem solving, where the decision maker is required to be at the real place -"Gemba" to observe the real situation -"Genjitsu" (Soares, Sousa, \& Nunes, 2012). Similarly in the Ohno circle, a decision maker is supposed to stand in a circle and observe a phenomenon to arrive at decisions by own free inquiry. Ngowi (2000) identifies independence as significant to $\mathrm{CI}$ and identifies it 
require both individual and group level autonomous initiatives rather than having to depend on the management.

Remarkable similarity is observed between autonomy in CI literature and Hongaku in Japanese Zen Buddhism. Hongaku conceptualize every being as already enlightened in some form (Waka, 1987). Simultaneously, the autonomy is based on the belief that all workmen are capable in organizational deliverables. Deming's belief system agrees with Hongaku as visible in quotes such as "quality is everybody's responsibility" and "quality is pride of workmanship".

\section{On Cause-Effect Validation}

Another difference visible in Buddhism and CI is on validation of root causes. Although both approaches are based on logic, the validation in CI have an if-then relationship whereas the validation in Buddhism have an if and only if relationship. A quality practitioner arriving at causes have to validate the possible causes by taking the order of the cause and effect, whereas in Buddhism this review occurs both ways (anuloma patiloma) from cause to effect (anuloma) and effect to cause (patiloma).

"The twelvefold application ...in its positive or direct aspect (anuloma) it makes known the causal chain behind suffering, demonstrating how the round of existence arises and turns through ... freely behind the shielding screen of ignorance. In its negative or reverse side (patiloma) it reveals the way to the cessation of suffering, showing that when ignorance is eliminated by the rise of true knowledge all the factors dependent on ignorance likewise draw to a close." (Bodhi, 1995)

The final outcome of this review is the truth of codependency of originations. Analyzing the forward and backward order of causes provide and only if relationship between causes. Thus, in the context of problem elimination there is forward and backward validity of existence.

\section{Discussion}

There are indications like some other Japanese concepts mentioned elsewhere in this article, that CI also facing adaptability issues related to culture. Bessant, et al. (2001) suggests that the experience of disappointment and failure with CI programs reported by many organizations is a result of lack of understanding of the behavioral dimension of CI. 
Simultaneously, it can be established that the many of the CI cycles such as PDCA, DMAIC, and RADAR were developed in a control perspective than a selfrealization perspective of process. For instance the Define- Measure-AnalyzeImprove and Control aspects of the DMAIC cycle are actually a controlling the CI process. Giving consideration to the behavioral aspect required for operationalization of incremental improvements such as kaizen, quality circles, suggestion schemes, quality improvement teams, cross functional activities, autonomous maintenance and design for six-sigma and innovation which are means of excelling in $\mathrm{CI}$, it should be noted that $\mathrm{CI}$ is least compatible in a control perspective.

On the other hand, all the models above discuss CI from rather an external point of view. They are external in its orientation in viewing the CI process apart from the process in which CI takes place. For instance the CI cycle of hypothesisexperiment- evaluation in the scientific method is external to the process in which the experiment takes place and is more like an ordered sequence of actions for the scientist. In other words these models distinguish CI process and the process to be improved as two different processes and try to address the CI process from the managerial control perspective. The issue in this frame of thinking is that it fails to recognize $\mathrm{CI}$ as inherent to the process and rather try to manage $\mathrm{CI}$ as a separate process. However it should be noted that by separating CI from the core process, not only it takes away what should rightly be inherent to that process but also it takes away improvement further away from $\mathrm{CI}$, as $\mathrm{CI}$ itself is a process for which PDCA is applicable. As the PDCA cycle of CI is external to the CI process, the CI process in principle is subjected to improvement through an external PDCA cycle which is not inherent to the CI process. This also leads to a bifurcation with respect to ownership of CI process and the actual process; owners of the CI process are the decision makers whereas the owners of the processes are only followers. Bessant, et al. (2001) states most organizations that operate on beliefs emerging from the scientific management approaches "sees a split into 'thinkers' and 'doers" which "implicitly opposes the CI values".

The implications of the control aspect is further evident in the learning cycle. CI requires incremental development with respect to organizational and personal knowledge. "CI is not just about systems - it is also about self-improvement and continual learning" (Poropat \& Kellett, 2006). However the organizations that practice $\mathrm{CI}$ "... assumes a binary split between having or not having CI, rather than seeing it as an emerging and learned pattern of behavior which evolves over time" (Bessant, Caffyn, \& Gallagher, 2001). 
The impact of not realizing full significance of the behavioral component of CI could provide barriers for these CI cycle principles to cultivate in sensitive organizational environments. However we surmise that originally the behavioral component was inherent even in the controlling cycle as can be seen in the Deming's 14 points for management. It provides insight to the thought process that follows in establishing the PDCA. These 14 points discuss largely the behavioral elements of PDCA which are by face value related to Buddhism. However in transition, PDCA seems to have loss its behavioral significance as evident in the previous research (Eg: Bessant, et al. (2001)).

\section{Conclusion}

There is a remarkable similarity between Buddhism and CI. Compiling the above findings together, it can be surmised that CI is evolved in a culture which is nourished by Buddhism.

With the Japans' rapid economic recovery and industrial success post World War II, its management model was benchmarked by many. For instance, the employee involvement \& engagement practices and Total Quality Management practices were tested by many organizations worldwide. However, in many cases these "Eastern" management models failed. Both Industries and academics first believed these are implementation failure, but later found otherwise. Today, it is a well-known fact that there are certain behavioral and cultural considerations associated with Japanese management styles which can lead to serious adaptability issues in many other organization cultures. In the case of CI, there is no difference.

Since now, the CI literature has not captured the exact issue surrounding the "cultural and behavioral" concern. As explained throughout the paper, CI is not really a new managerial experience for Japan as its organization culture was molded with Buddhism in background. The practices, methods are behaviors associated were nothing foreign to their original set of values.

Buddhism qualifies as a binding agent between the different aspects lies within the purview of CI. As such, CI can have better adoptability in organizations that are connected with Buddhism. On macro level, as a Buddhist country, the local economy may benefit from the adaptation of CI. 


\section{Bibliography}

Albahari, M. (2006). Analytical Buddhism : the two-tiered illusion of self. Basingstoke, New York: Palgrave Macmillan.

Bessant, J., Caffyn, S., \& Gallagher, M. (2001). An evolutionary model of continuous improvement behaviour. Technovation, 67-77.

Besterfield, D. H., Besterfield-Michna, C., \& Besterfield-Scare, M. (2003). Total Quality Management (3rd ed.). New Jersy: Person Education Inc.

Bhuiyan, N., \& Baghel, A. (2005). An overview of continuous improvement: from past to present. Management Decision, 43(5), 761-771.

Bjurström, E. (2012). Minding the contexts of mindfulness in quality management. International Journal of Quality \& Reliability Management, 29(6), 669-713.

Bodhi. (1995). Transcendental Dependent Arising:A Translation and Exposition of the Upanisa Sutta. Retrieved from Access to Insight: http://www.accesstoinsight.org/lib/authors/bodhi/wheel277.html .

CQI Technical Assitance Training Manual. (2010). PDCA Method Enhanced: the Seven Step Model of CQI Beyond Rapid Cycle Improvement. Raleigh, NC: Southeastern Kidney Council.

Deming, W. E. (1994). The new economics: for industry, government, education (second ed.). Mass: Cambridge.

Evans, T. J. (1996). Deming's system of profound knowledge: An overview for international educators. The Escuela Internacional Sampedrana. San Pedro Sula, Honduras.

Fletcher, L., \& Hayes, S. C. (2005). Relational frame theory, acceptance and commitment therapy, and a functional analytic definition of mindfulness. Journal of RationalEmotive \& Cognitive-Behavior Therapy, 23(4), 315-336.

IGNOU. (2001). MS 96 Total Quality Management. New Delhi: Aravali.

Lamberton, G. (2005). Sustainable Sufficiency - an Internally Consistent Version of Sustainability. Sustainable Development, 13, 53-68.

Marksberry, P., Badurdeen, F., Gregory, B., \& Kreafle, K. (2010). Management directed kaizen:Toyota's Jishuken process for management development. Journal of Manufacturing Technology Management, 21(6), 670-686.

Ngowi, A. (2000). Impact on culture on the application of TQM in the construction industry in Bostwana. International Journal of Quality and Reliability Management, 17(4/5), 442-452.

Oakland, J. S. (2004). Oakland on Quality Management. (Butterworth-Heinemann, Ed.) Oxford Boston: Elsevier. 
Ohno, T. (1988). Toyota production system : beyond large-scale production. Cambridge, Mass: Productivity press.

Piyadassi. (2008). Dependent Origination. Kandy, Sri Lanka: Buddhist Publication Society.

Poropat, A., \& Kellett, J. (2006). Buddhism and TQM: An alternative explanation of Japan's adoption of Total Quality Management. Proccedings of the 20th ANZAM conference,. Austrailian and New Zeeland Academy of Management.

Radawski, D. (1999). Continuous Quality Improvement: Origins,Concepts, Problems, and Applications. Perspective on Physician Assistant Education, 10(1), 12-16.

Radric, C. A. (2007). Enlightened Management: An Analysis of Buddhist Precepts Applied to to Managerial Activity. Journal of Global Business Management.

Rettig, M., \& Simons, G. (1993). A project planning and development process for small teams. Communications of the ACM, 36(10), 45-55.

Schumacher, E. (1973). In Small is Beautiful: A Study of Economics as if People Mattered. New York: Harper \& Row.

Smith, G. F. (2000, April). Too many types of quality problems. Quality Progress, 43-49.

Soares, J., Sousa, S., \& Nunes, E. (2012). Application of three realities approach to customer complaint handling in motorcycle industry. International conference on industrial engineering and operations management. Guimarães,, Portugal: Associação Brasileira de Engenharia de Produção.

Sokovic, M., Jovanovic, J., Krivokapic, Z., \& Vujovic, A. (2009). Basic Quality Tools in Continuous Improvement Process. Strojniški vestnik - Journal of Mechanical Engineering, 55(5), 333-341.

Sokovic, M., Pavletic, D., \& Pipan, K. K. (2010). Quality improvement methodologiesPDCA cycle, RADAR matrix, DMAIC and DFSS. Journal of Achieevments in Materials and Manufacturing Engineering, 476-483.

Sumedho, A. (1992). The Four Noble Truths. Hertfordshire: Amaravathi Publications.

Tsering, T., \& McDougall, G. (2005). The four noble truths. Boston, Mass: Wisdom Publications.

Waka, S. (1987). Inherent Enlightenment (hongaku shiso) and Saich's Acceptance of the Bodhisattva Precepts. Japanese Journal of Religious Studies, 14(2-3), 113-127.

Yokosawa, K., \& Steenhuis, H.-J. (2012). Influence of national level factors on international kaizen transfer:An exploratory study in Netherlands. Tokyo: Manufacturing management research center, University of Tokyo.

Zangwill, W. I., \& Kanton, P. B. (1998, Jul.). Towards a theory of continual improvement and the learning curve. Management Science, 44(7), 910-920. 